\title{
Traumatic Subcutaneous Emphysema
}

National Cancer Institute

\section{Source}

National Cancer Institute. Traumatic Subcutaneous Emphysema. NCI Thesaurus. Code C35082.

The infiltration of air into the skin tissue secondary to traumatic tissue disruption. 